\title{
Test of Beyond-Standard-Model Scenarios with Low Threshold Germanium Detectors
}

\author{
Lakhwinder Singh*† \\ Institute of Physics, Academia Sinica, Taipei 11529, Taiwan \\ E-mail: lakhwinder@gate.sinica.edu.tw
}

\begin{abstract}
The existence of physics beyond the standard model has been concretely established based on the observation of neutrino flavor oscillations and it has open many questions about the neutrino's intrinsic properties, including the absolute mass, Majorana or Dirac nature and the relative mass hierarchy of the neutrino. Germanium $(\mathrm{Ge})$ ionization detectors with their unique features and diverse applications in fundamental research are novel candidates for such a search. The TEXONO Collaboration has been pursuing the research program on low energy neutrino interactions and light WIMP Dark Matter with Ge-ionization detectors at the Kuo-Sheng Neutrino Laboratory (KSNL) in Taiwan and in the China Jinping Underground Laboratory (CJPL) in China. We present highlights the potentials of Ge-detectors for the studies of active neutrino electromagnetic interactions, light WIMP, sterile magnetic moment and neutrino-nucleus coherent scattering.
\end{abstract}

The 39th International Conference on High Energy Physics (ICHEP2018)

4-11 July, 2018

Seoul, Korea

*Speaker.

${ }^{\dagger}$ on behalf of the TEXONO Collaboration 


\section{Introduction}

The standard model (SM) of particle physics describes the all known particles and their interaction (ignoring gravity) to a remarkable degree of precision. The discovery of the Higgs Boson has completed the set of particles predicted by SM. However, there are still several big questions remain that SM does not address, such as the observed matter-antimatter asymmetry in the Universe, the non-zero neutrino masses and mixing, and the existence of dark matter. Experimental verification of the existence of neutrino masses and mixing have provided the first direct evidence of physics beyond the SM, which has triggered intensive studies of nontrivial electromagnetic properties in both theoretical and experimental framework.

Germanium (Ge) ionization detectors [1] with their low intrinsic radioactivity background, excellent energy resolution, and sub-keV threshold are the best candidates to study physics beyond the SM, including the neutrino electromagnetic properties, neutrino-nucleus coherent scattering with reactor neutrinos, WIMP and axion dark matter searches, and other exotic particle physics phenomena $[2,3,4]$.

\section{Electromagnetic Properties of Neutrino}

The electromagnetic properties of neutrino is an interesting venue to look for new physics beyond the SM. The massive neutrinos acquire non-vanishing tiny charge radii squared, magnetic dipole moments, anapole moments (require parity violation in addition), and electric dipole moments (require both parity and time-reversal violation in addition) through electroweak radiative corrections. The current experimental limits on some electromagnetic properties of neutrino are orders of magnitude larger than the SM predictions, this is a hint for new physics beyond the SM.

The elastic neutrino-electron $(v-$ e) scattering is a most sensitive process to study neutrino magnetic moments $\mu_{v}$ [5] and neutrino milli-charge $q_{v}$ [6] at low energy regime due to $1 / \mathrm{T}$ and $1 / \mathrm{T}^{2}$-dependence of the differential rate, respectively. When energy transfer in neutrino scattering starts to overlap with atomic scales, atomic effects play significant roles to the cross sections. We adopted the Multi-Configuration Relativistic Random-Phase Approximation theory, to calculate the $\mu_{v}$ and $q_{v}$ induce differential cross sections. This ab-initio many-body theory also provides the better description for the structure of Ge atoms as well as photo-absorption data of Ge crystal at photon energy $\geq 80 \mathrm{eV}$ [7]. The upper limits of $q_{v}<2.1 \times 10^{-12}$ and $\mu_{v}<2.6 \times 10^{-10} \mu_{B}$ are derived using PCGe (point contact Ge-detector) 124.2(70.3) kg-days of ON (OFF) data at $90 \%$ C.L. The upper bounds on $q_{v}$ and $\mu_{v}$ are limited due to low statistics and high background in sub$\mathrm{keV}$ region. Our best experimental upper bounds $\mu_{v}<7.4 \times 10^{-11} \mu_{B}$ at $90 \% \mathrm{CL}$ is derived from 570.7(127.8) kg-days of ON (OFF) with $12 \mathrm{keV}$ threshold data.

\section{Light Dark Matter Searches}

It is now well established that about one-quarter of the energy density in the Universe is composed of cold dark matter. Its non-gravitational interactions with normal matter are still unknown. Weakly Interacting Massive Particles (WIMP) is a generic class of cold dark matter. A number of direct detection experiments are pursuing the intensive research programs towards sensitivity to 
light WIMP mass region $\mathrm{m}_{\chi}<10 \mathrm{GeV}$. Based on data taken with CDEX-10 experiment $(10 \mathrm{~kg}$ p-type point contact Ge-detector array immersed in liquid nitrogen) at CJPL [4] improved limits are derived for mass WIMP.

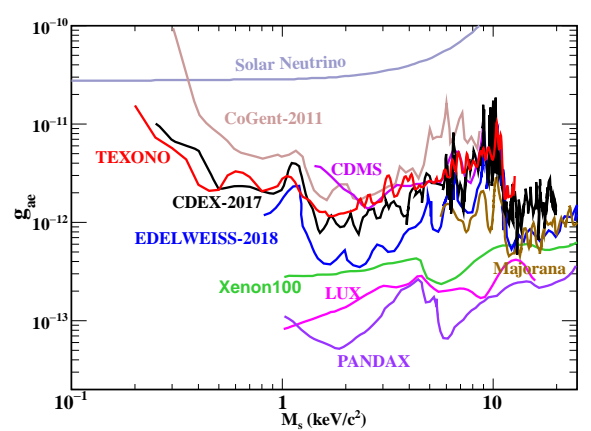

(a)

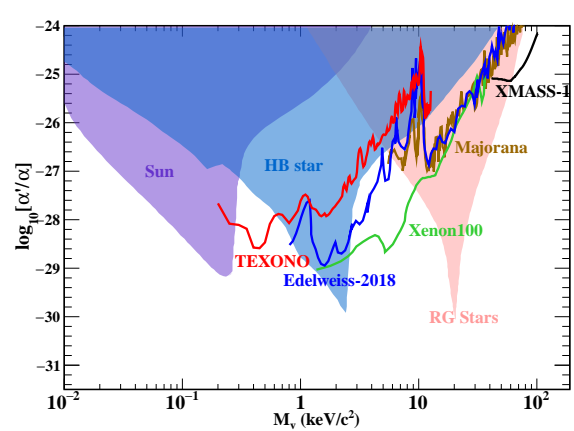

(b)

Figure 1: (a) Limits on the coupling of pseudoscalar super-WIMP with electron as function of mass from various benchmark experiments at $90 \%$ C.L. (b) The $90 \%$ C. L. bounds on vector bosonic dark matter coupling from different astrophysical sources as well as other benchmark experiments.

Bosonic super-WIMP is well-motivated class of dark matter with coupling smaller than the weak scale. These particles are experimentally very interesting due to their absorption via ionization or excitation of an electron in the target-atom of the detector. Bosonic super-WIMP would deposit energy equivalent to their rest mass, which manifest as a photo-peak. Therefore, a good energy resolution device like PGe-detectors have advantages to study such class of DM. Using the low-background and low-threshold data from KNSL with PGe detectors, improved limits at $90 \%$ $\mathrm{CL}$ on the coupling constant of pseudo-scalar $\left(g_{\text {aee }}\right)$ and vector super-WIMP $\left(\alpha^{\prime} / \alpha\right)$ as a function of particle mass are derived with the assumption that the bosonic DM constitutes all of the galactic DM with local DM density $\rho_{\chi}=0.3 \mathrm{GeV} / \mathrm{cm}^{3}$. The upper bounds on coupling constant $g_{\text {aee }}$ and $\alpha^{\prime} / \alpha$ at various mass are shown in Fig. 1(a) and Fig. 1(b), respectively.

\section{Dark Matter Sterile Neutrino Search}

Sterile neutrinos are singlets in the SM gauge groups, and they are described via their mass $\left(\mathrm{m}_{s a}\right)$ and active-sterile mixing amplitude. Their masses, mixing angles and couplings are unknown. They are in addition viable candidates of dark matter. The massive sterile neutrino can undergo radiative decays via mixing with the active SM neutrino. This process is considered as one of the golden modes to look for keV-scale sterile neutrino. It is recently identified that inelastic scattering process for a non-relativistic sterile neutrino has a pronounced enhancement in the differential cross section at energy transfer about half of its mass, as illustrated in Fig. 2(a). This feature would manifest itself as peaks in the measurable energy spectra [8]. The constraints on transition magnetic moment of sterile neutrino $\left(\mu_{v_{s a}}\right)$ are derived from data taken with low-background and low-threshold PCGe detectors at KSNL, as shown in Fig. 2(b). These constraints on $\mu_{v_{s a}}$ are more stringent than the current direct upper limits of active neutrinos $\mu_{v}$, due to pole structure in differential cross section at half of its mass where $q^{2} \rightarrow 0$.

Ge-detectors with sub-keV threshold have been used to study neutrino properties and interactions, neutrino-nucleus coherent scattering, WIMP and super-WIMP searches. Data taking and 


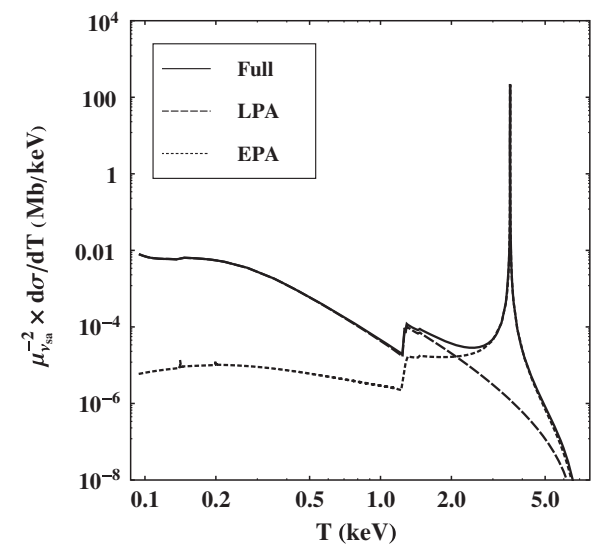

(a)

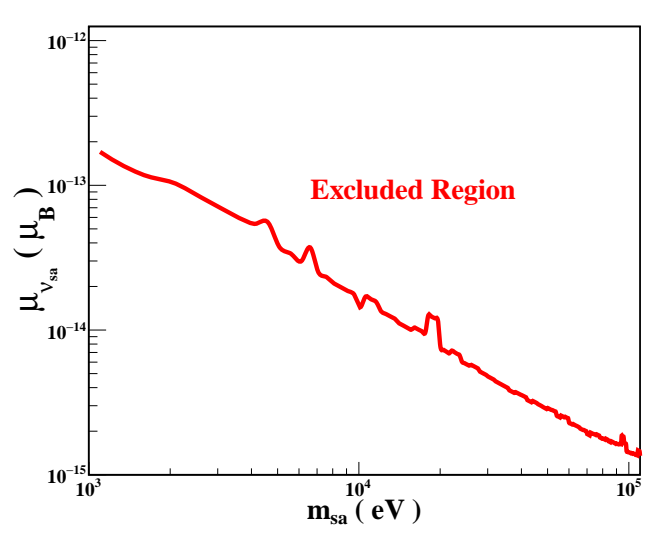

(b)

Figure 2: (a) The differential scattering cross section of nonrelativistic sterile neutrinos and germanium atoms through the transition magnetic moment $\mu_{v_{s a}}$ with $\mathrm{m}_{s a}=7.1 \mathrm{keV}$ and $\mathrm{E}_{v}=1 \mathrm{MeV}$. For comparison, the results of LPA (longitudinal photon approximation) and equivalent photon approximation (EPA) are also shown, and (b) Exclusion curve of $\mu_{v_{s a}}$ at $90 \%$ C.L. from KSNL data.

analysis are continuing at KSNL, complemented by the CDEX experiment at CJPL on WIMP and axion-like dark matter searches. Intensive $R \& D$ programs are being pursued to understand and suppress the sub-keV background.

\section{References}

[1] TEXONO collaboration, A. K. Soma et al., Characterization and Performance of Germanium Detectors with sub-keV Sensitivities for Neutrino and Dark Matter Experiments, Nucl. Instrum. Meth. A836 (2016) 67 [1411.4802].

[2] H. T. Wong, Taiwan EXperiment On NeutrinO - History and Prospects, Int. J. Mod. Phys. A 33 (2015) 1830014 [1608.00306].

[3] J.-W. Chen et al., Constraining neutrino electromagnetic properties by germanium detectors, Phys. Rev. D 91 (2015) 013005 [1411.0574].

[4] CDEX Collaboration collaboration, H. Jiang et al., Limits on light weakly interacting massive particles from the first $102.8 \mathrm{~kg} \times$ day data of the cdex-10 experiment, Phys. Rev. Lett. 120 (2018) $241301[1802.09016]$.

[5] TEXONO Collaboration collaboration, H. T. Wong et al., Search of neutrino magnetic moments with a high-purity germanium detector at the kuo-sheng nuclear power station, Phys. Rev. D 75 (2007) 012001 [hep-ex/0605006].

[6] J.-W. Chen et al., Constraints on millicharged neutrinos via analysis of data from atomic ionizations with germanium detectors at sub-kev sensitivities, Phys. Rev. D 90 (2014) 011301 [1405. 7168].

[7] J.-W. Chen et al., Atomic ionization of germanium by neutrinos from an ab initio approach, Phys. Lett. B 731 (2014) 159 [1311.5294].

[8] J.-W. Chen et al., Atomic ionization by sterile-to-active neutrino conversion and constraints on dark matter sterile neutrinos with germanium detectors, Phys. Rev. D 93 (2016) 093012 [1601.07257]. 